\title{
Pinched Material Einstein Space-Time Produces Accelerated Cosmic Expansion
}

\author{
M. S. El Naschie \\ Department of Physics, Faculty of Science, University of Alexandria, Alexandria, Egypt \\ Email: Chaossf@aol.com
}

Received 7 January 2014; revised 5 February 2014; accepted 13 February 2014

Copyright @ 2014 by author and Scientific Research Publishing Inc.

This work is licensed under the Creative Commons Attribution International License (CC BY). http://creativecommons.org/licenses/by/4.0/

(c) (i) Open Access

\begin{abstract}
An instructive analogy between the deformation of a pinched elastic cylindrical shell and the anti-gravity behind accelerated cosmic expansion is established. Subsequently the entire model is interpreted in terms of a hyperbolic fractal Rindler space-time leading to the same robust results regarding real energy and dark energy being $4.5 \%$ and $95.5 \%$ respectively in full agreement with all recent cosmological measurements.
\end{abstract}

\section{Keywords}

Dark Energy; Rindler Space-Time; Cartan Torsion; Pinched Elastic Shells; Negative Gravity; Cosmic Accelerated Expansion; Fractal Space-Time; Topological Defects; Hardy's Quantum Entanglement; Hawking's Radiation; Cosserat Elasticity

\section{Introduction}

When a long elastic cylindrical shell is pinched in the middle, it deforms in the following slightly contra- intuitive way. Locally and in the vicinity of the pinching, the circular cross-section deforms and changes its curvature from a circular to an oval shaped one. With increasing distance from the pinching force and towards the edges the oval cross-section rotates until it becomes perpendicular to the oval at the pinched middle of the cylinder. Such geometrical deformation is only possible because of the "material" nature of the cylinder and could not make sense except for the continuum mechanics of a tangible material surface as opposed to an idealized purely figmental geometrical non-materialistic space such as those of Euclid or Riemann. Cylindrical shells like all real structures are endowed with complex shear and torsional forces as is well known from the theory of elasticity, plasticity and Rheology. Likening the local curvature in the pinched region with a positive attractive gravity pulling things together we are reasonably justified to liken the perpendicular curvature at the extremity of the cylinder with a negative repulsive gravity which pushes things apart. 
In the present work this simple analogy is taken literally and pushed to its ultimate by imaging the whole set up taking place in four dimensional spaces. To tame the involved "infinitely" long 4D "quasi cylinder" we use the sophistication of hyperbolic geometry and utilize the Poincare-Beltrami projection to establish a connection to a Penrose-like fractal tiling universe. It is then not particularly difficult to imagine what one will discover next when connecting each of the ramified fractal tiles to a hyperbolic fractal Rindler space resembling a black hole or a knot compliment at infinity. At the circular horizon of the Poincare-Beltrami projection and taking the isomorphic length into consideration, each fractal point is a head of a Rindler wedge. In turn the wedge consists of two parts, a hyperbolic triangle with a "topological" area equal to $\phi^{5} / 2$ where $\phi=(\sqrt{5}-1) / 2$ and a circular segment shape joined to the triangle with an area or rather a topological measure equal to $1-\left(\phi^{5} / 2\right)=5 \phi^{2} / 2$. Subsequently we use various facts connected to the thermodynamically interpretation of gravity, Hawking's radiation, non-commutative geometry, Cantorian E-infinity theory as well as the algebraic topology theory of cosmic defects to reason that $\phi^{5} / 2$ gives us the COBE, WMAP and Planck measured $4.5 \%$ energy density of the cosmos $E(0)=\left(\phi^{5} / 2\right)\left(m c^{2}\right) \simeq m c^{2} / 22$ while $5 \phi^{2} / 2$ is the $95.5 \%$ factor of the "missing" dark energy density $E(D)=\left(5 \phi^{2} / 2\right)\left(m c^{2}\right)$ corresponding to the antigravity effect behind the observed accelerated expansion of the cosmos. In this sense and by setting space, time and matter truly on the very same footing we could loosely say that attractive gravity pinches the "material" space-time counterpart of Einstein's gravity and produces the observed puzzling anti-gravity accelerating expansion of the cosmos. In fact it is natural to have negative curvature and thus negative gravity in a Cantorian-fractal space-time where there are no real points at all and therefore torsion does not vanish by taking the deceptive limit of a fundamentally granular space-time setting. We conclude by noting that a cosmological constant $\Lambda=-1$, a topological empty set dimension $D_{T}=1$, negative curvature at a horizon or a conjectured negative dimension of a texture-topological defect are all basically tautological statements saying essentially the same thing, namely that there is a cosmic accelerated expansion and that dark energy is what stands behind this negative gravity force.

\section{Preliminary General Remarks}

A universal wisdom that has been well tested over the years is that in science as in life, asking the right question is almost half of the answer [1]. To put it less delicately, it is almost universally agreed that asking a stupid question invariably results in a stupid answer. It seems that a few scientists were more equipped to ask the right question than most of us and this is the main philosophy probing the present paper [1]-[14]. Hermann Weyl's famous book "Raum, Zeit, Materie" may be as good a starting point as any [2]. Do we really treat space, time and matter in a democratic way? Sure enough space and time were fused by the Minkowsky-Einstein program however nothing similar was systematically undertaken with the same vigour [3]-[5]. For instance the geometry of space-time used in physics is nowhere as "real" as the geometry used in say the theory of elasticity or plasticity. Of course there are many models used in relativistic quantum physics which utilized hydrodynamical paradigms and even modified fluid mechanics equations but these important efforts are relatively the exception and do not go as far as one could imagine. To put space-time and matter on the same footing requires a new material-like geometry [6]-[28] with granular structure for which the torsional part of the connection [24] does not vanish.

In the present work we advocate the idea that such geometry exists since a relatively long time and that it is a generalization of what E. Cartan and the brothers Cosserat developed in 1909 when married to modern Cantorian fractals [7] [8] [12] [14]. In fact we will show various completely unsuspected relations between metal forming engineering problems and the negative pressure behind the observed unexpected acceleration rather than deceleration of cosmic expansion [15]-[17]. To put it succinctly in a few sentences, we will show that anti-gravity is essentially the same phenomena as anti-curvature of a pinched long cylindrical shell once this cylinder is put in the projective hyperbolic plane corresponding to 4 and 5 dimensional fractal space-time. The present work is thus a monolithic synthesis of the work of Einstein, Cartan, Hawking, Rindler, Penrose, the Cosserat brothers, Conne, Unruh and the school of fractal Cantorian space-time to mention only a few of the main sources pouring into our present theory [15]-[78].

\section{Keeping an Open Mind about the Fractal-Thermodynamical Fluctuation Origin of Gravity}

It is important that we point out from the outset a few fundamental points which represent some departure from 
the orthodoxy of general relativity. In short this requires what we consider a minimum of open mindedness about the following concepts and experimental findings:

3.1. As in Feynman's conjecture extended by the author we view gravity as the effect of the passing of fractal time [7] [8] [21] [22].

3.2. Hawking's radiation, Rindler's wedge and Unruh's temperature are backed by real physics and are by no means mathematical artifacts [3] [9] [17] [20].

3.3. Hardy's quantum entanglement is real and was experimentally verified [24]. The golden mean to the power of five first found by Hardy as a quantum probability and recognized as such by the author is profound [19] [22].

3.4. The COBE, WMAP and Planck measurements as well as other recent astronomical as well as astrophysical anomalies are real and will not be dismissed here as misinterpretation, faulty calculations or defects of electronic equipment [17] [20].

At this point it is appropriate to note the work of Padmanabhan [32] [33] as one of the main guiding lights in uncovering the thermodynamical roots of gravity. On the other hand our hyperbolic geometrical fractal conception of space-time [79] is also at the root of thermodynamics itself as is obvious from the thermal character of Unruh's temperature [9] [17] [27]. The same view point applies of course to electromagnetism where we are justified in seeing $\bar{\alpha}_{o} \cong 137$ as by far more fundamental than Newton's constant, the speed of light or Planck's constant [7] [8] [12] [17] [21] [23].

\section{Elementary Derivation of Einstein's Revised Formula for Ordinary Energy $E \simeq m c^{2} / 22$}

Needless to say, when Einstein drove his famous $E=m c^{2}$ he did not write a Lagrangian. However, supposed he knew how to do it in what with current prejudice is the only acceptable way forward, namely writing down a Lagrangian [24]. First such a Lagrangian would lead in our opinion to a few realizations [12] [15] [18] [22]. For a start the only degree of freedom from a particle physics view point would be the only messenger particle known at the time of Einstein, namely the photon. Energy on the other hand would be the Eigenvalue of a Schrödinger equation however not that of a particle but rather that of the entire universe [24]. Thus E of Einstein would be the Eigenvalue of an unknown quantum gravity Schrödinger-like equation. However, we know in the meantime that the physics of our universe is best approximated by at least 12 photon-like particles and not only one photon. On the other hand we know very well that reducing a 12 degrees of freedom Lagrangian to only one degree of freedom Lagrangian would lead to a gross over estimation of the corresponding Eigenvalue, i.e. the energy $E$ of Einstein which is a well known theorem by Lord Rayleigh [25]. Thus we see that $E=m c^{2}$ is much larger than what a locally 12 degrees of freedom Lagrangian allows. So much for the qualitative situation, the quantitative one is more involved. However, an educated guess leads to the following line of reasoning. Noting that the kinetic energy of Newton $E_{N}=(1 / 2) m v^{2}$ and $E$ of Einstein differs mainly by a "scaling" factor $(1 / 2)$ when disregarding the limit $v \rightarrow c$ and noting also that self similarity is a fundamental aspect of both the macro (solar system) and the micro cosmos (Bohr atom) then one is encouraged to think that $E=m c^{2}$ could be scaled down proportionately to $E=m c^{2} / 22$, where $1 / 22$ is the scaling factor [26]-[28]. The value 22 could be thought of in two different obvious ways. It is the 26 bosonic dimensions of the Veneziano space-time minus Einstein's 4 di- mensional space-time, i.e. [15] [16] [27]

$$
\lambda=1 /(26-4)=1 / 22 \text {. }
$$

Alternatively we use $E_{N}=\frac{1}{2} m v^{2}$ and invoke the scaling [15] [27]

$$
\bar{\lambda}=1 /[|S U(3) S U(2) U(1)|-\gamma]=1 /(12-1)=1 / 11
$$

and the limit $v \rightarrow c$ to find that [27]

$$
E_{N} \rightarrow E(O)=\bar{\lambda}\left(\frac{1}{2} m(v \rightarrow c)^{2}\right)=\left(\frac{1}{11}\right)\left(\frac{1}{2}\right) m c^{2}=\frac{1}{22} m c^{2}
$$

The preceding plausibility derivation could be made mathematically water tight in various ways discussed in previous publications and will not be followed here any further in order not to lose the main thread of the pre- 
sent work and its objective [17]-[20].

\section{Details of Rindler Space Calculations Leading to the Areas $\phi^{5} / 2$ and $5 \phi^{2} / 2$}

We follow Figure 1 representing a Rindler space and the associated horizon [29] [30]. In this section we concentrate on the questions pertaining to measure theory, i.e. the various Lorentzian invariant hyperbolic areas and for the moment relegate the question of physical interpretation to a back seat. We see that we have three distinct areas. The first $A_{o}$ is the total area of the large triangle 1,2, 3. Calculating the area of $A_{o}$ is truly trivial since it consists of two symmetric triangles leading to

$$
A_{o}=2\left[\frac{1}{2} t(\eta / 2) Z(\eta / 2)\right]
$$

where $\eta$ is the opening angle of the Rindler wedge. Since

$$
t(\eta)=\ell \sinh (\eta)
$$

and

we can write $A_{o}$ as

$$
Z(\eta)=\ell \cosh (\eta)
$$

$$
A_{o}=\ell^{2} \cosh (\eta / 2) \sinh (\eta / 2)
$$

where $\ell=1 / a$ is the distance between the Rindler horizon and the observer as seen by him and a is the con-

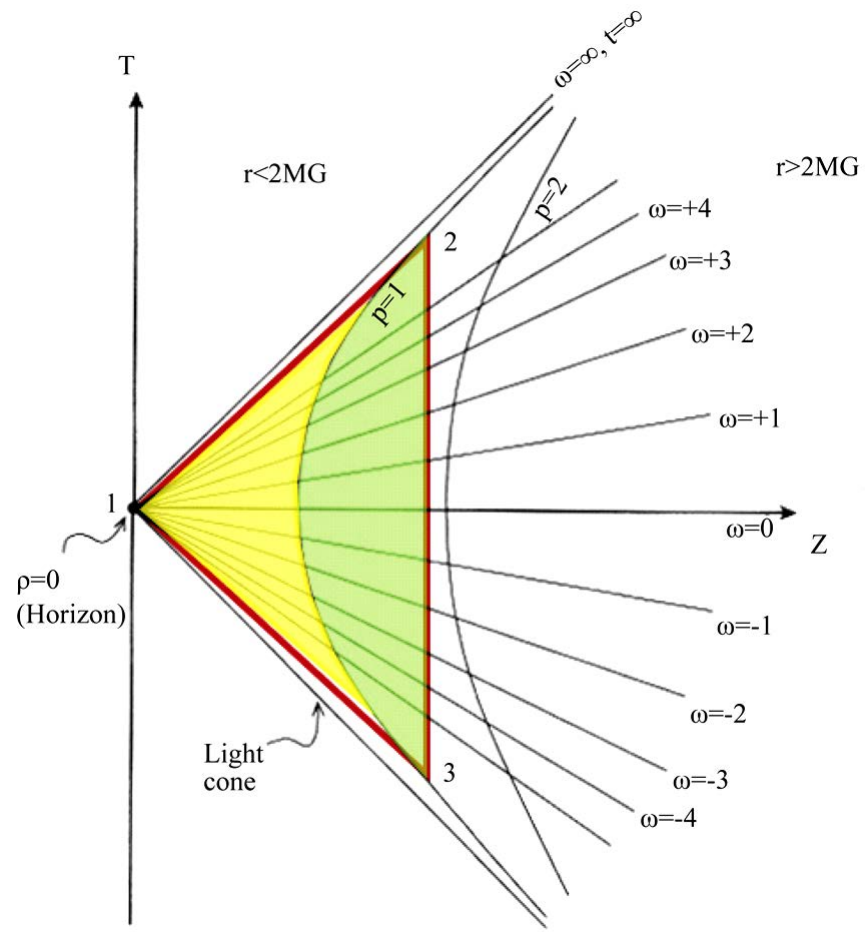

Figure 1. Equal time and proper distance surfaces in Rindler space [9] [17] [29] [30]. Note that the area of the triangle $(1,2,3)$ is Lorentz invariant and is given by $A=[T(w / 2) z(w / 2)]$. In addition the area of the hyperbolic light yellow triangle is equal to the topological ordinary energy $\left(\phi^{5} / 2\right)$ while the area of the green circular segment is equal to the topological dark energy $\left(5 \phi^{2} / 2\right)$ where $\phi=2 /(\sqrt{5}+1)$. The actual calculation is given in the main text and depends upon simple integration of hyperbolic trigonometrically functions [31] [79]. 
stant Rindler acceleration [9] [17] [29] [30].

The second area is the symmetric hyperbolic segment $A_{1}$. This gives twice the integral of half of the segment as

$$
A_{1}=2\left[\ell^{2} \sinh (\eta / 2) \cosh (\eta / 2)-\eta / 2 \ell^{2}\right]=5 \phi^{2} / 2
$$

Since

$$
\mathrm{Z}(\eta)=\ell \cosh (\eta)
$$

Then

$$
\frac{\mathrm{d} z(\eta)}{\mathrm{d} \eta}=\sinh (\eta)
$$

and therefore

$$
\mathrm{d} z(\eta)=\ell \sinh (\eta) \mathrm{d}(\eta) .
$$

Inserting one finds

$$
A_{1}=2 \int_{0}^{\eta / 2} t(\eta) \mathrm{d} z(\eta)=2 \int_{0}^{\eta / 2} \ell \sinh (\eta) \ell \sinh (\eta) \mathrm{d} \eta=2 \ell^{2} \int_{0}^{\eta / 2} \sinh ^{2}(\eta) \mathrm{d} \eta .
$$

This is a straight forward simple integration but could also be found in any standard handbook of mathematics to be [31]

$$
\begin{aligned}
A_{1} & =2 \ell^{2}\left[\frac{1}{2} \sinh \eta \cosh \eta-\frac{1}{2} \eta\right]_{0}^{\eta / 2}=2 \ell^{2}[\sinh \eta \cosh \eta-\eta]_{0}^{\eta / 2} \\
& =2 \ell^{2}\left[(\sin \eta / 2)(\cos \eta / 2)-(\eta / 2)\left(\ell^{2}\right)\right] .
\end{aligned}
$$

Finally the area of the hyperbolic triangle $A_{2}$ is found simply as the difference between $A_{0}$ and $A_{1}$ (see Figure 1). Consequently

$$
A_{2}=A_{o}-A_{1}=\ell^{2} \cosh (\eta / 2) \sinh (\eta / 2)-\left[\ell^{2} \sinh (\eta / 2) \cosh (\eta / 2)-\eta / 2 \ell^{2}\right]=\left(\ell^{2} \eta\right) / 2 .
$$

It is one of the fundamental results of the unit interval "topological" physics introduced in earlier work that $c=\phi$ and $m=\phi^{3}$ [15] [36] [77]. On the other hand, a few moments of deep reflection will reveal that $m=\eta$ and $c=\ell$. Consequently

$$
\eta \simeq \phi^{3} \text { and } \ell=\phi
$$

Inserting in $A_{2}$ one finds

$$
A_{2}=\phi^{5} / 2 .
$$

That immediately leads to our second most important result, namely [9] [17] [27]

$$
A_{1}=5 \phi^{2} / 2
$$

where $\phi=(\sqrt{5}-1) / 2$.

\section{Pinching Space-Time}

Various experiments with pinched elastic and elastoplastic cylindrical shells were actually performed long ago by the late Prof. W. Johnson (see Figure 2), when he headed his laboratory in Cambridge and before that in Manchester [74]. In fact it is extremely easy to demonstrate the effects of induced local change of curvature causing a considerable distance away a change of curvature of opposite sign [15]. For that we need nothing more than a large sheet of writing paper rolled into a long cylinder and squeeze it in the middle as described in previous work [15] [22] [23]. That way we establish at a minimum an analogy connecting not only engineering metal forming with cosmology but also with thermodynamics [3] [32].

\section{Self Similarity, P-Adic Quantum Physics and Cantorian Space-Time}

Integers are possibly the simples source of self similarity in physics. A trillion is nothing but unity scaled up a 


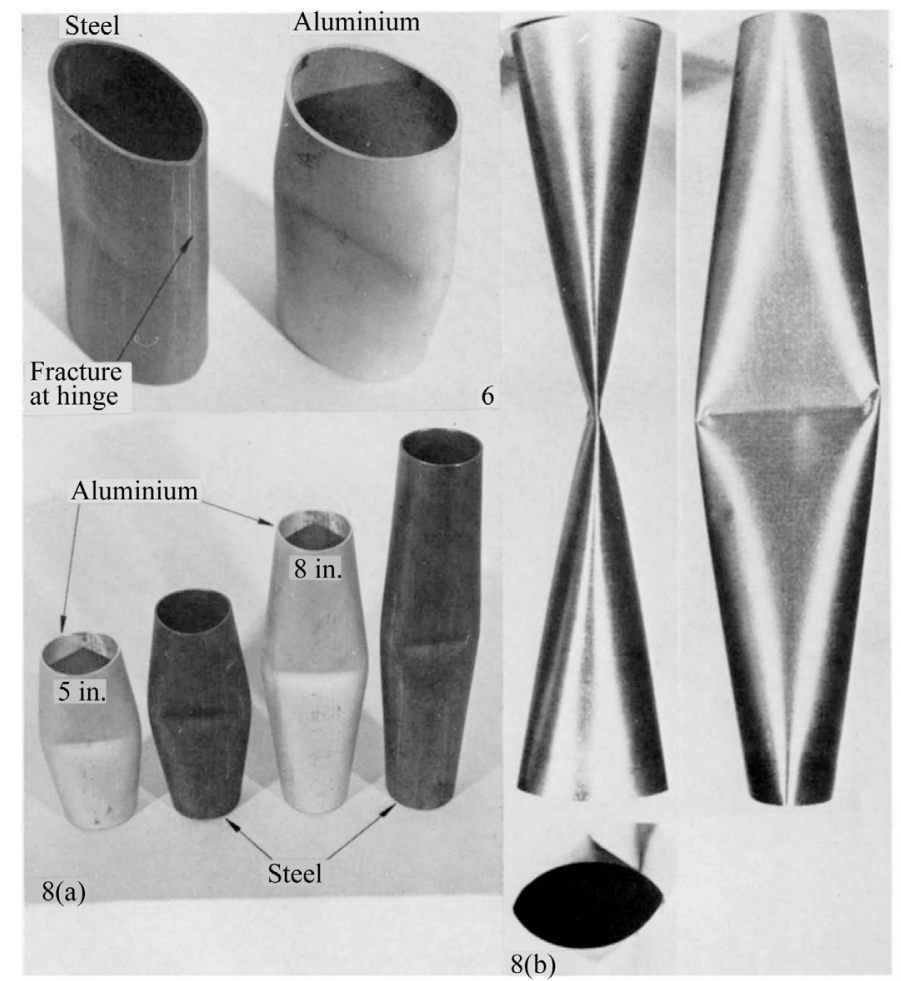

Figure 2. Actual experiments with elastic and plastic cylindrical shells [74]. The deformation of the pinched cylinder provides an instructive insight into the difference between real material space and abstract mathematical idealization of space. In a real space a local change of curvature at the middle of the pinched cylinder induces a seemingly opposite change of curvature at the edges of the shells. We imagine the situation in $4 \mathrm{D}$ space to be a higher dimensional analogue to gravity and anti-gravity. A simple paper model demonstrating the above Cosserat-like behaviour was given by the author in an earlier publication of the present journal [15].

trillion times. Number theory is of course very close to the continuum hypothesis and consequently the most fundamental question regarding the nature of space and time [7] [8] [21] [22]. It is therefore important to understand the intimate relation of the present paper with the fundamental result found by the school of P-Adic quantum physics [37]-[43] which we discussed in some details on previous occasions. We stress that only zero and infinity are not ordinary numbers but deep mathematical-philosophical concept. Since unity differs only by a scaling factor we see the fundamental meaning of the unit interval physics where the speed of light is a natural topological quantity $c=\phi \quad[9][15][16][23]$.

\section{Dark Energy and Dark Matter Segregated and Unified}

On the most fundamental level of transfinite set theory we have only the ordinary energy connected to the quantum zero set particle, i.e. $E(O)=\left(\phi^{5} / 2\right) m c^{2}$ which is directly proportional to the area of the hyperbolic traingle of the Rindler wedge $A_{2}$ and the dark energy connected to the quantum empty set of the wave proportional to the circular segment area $A_{1}=1-A_{2}=5 \phi^{2} / 2$ (see Figure 1). Clearly not all of $A_{1}$ dark energy is pure energy but some of it is dark matter exactly as part of the ordinary energy is ordinary matter expressed in terms of energy following the theoretical insight of Einstein and the essence of his formula if not its exact quantitative prediction which needed the present revision. We are not yet in a position to give a stringent mathematical distinction between dark energy and dark matter, which although lumped together in the energy of the five dimensional empty set theory, has different physical effects and manifestation. However, what we can do here is to give a logically coherent plausibility explanation converging towards a mathematical water tight explanation for the 
difference between dark energy and dark matter [27].

Let us recall first that our previous calculations demonstrated that while $4.5 \%$ of the energy density of the cosmos is measurable ordinary energy and matter, the rest, i.e. $100-4.5=95.5 \%$ of the energy density must be in the form of dark energy which we believe to be responsible for the initially surprising astrophysical observations connected to the accelerating cosmic expansion in addition to dark matter which we presume to be responsible for various astronomical anamolous observations [24] [32]. Let us further recall that our fundamental equation from which we construct our most fundamental coupling constant, namely $\bar{\alpha}_{0} \cong 137$ is found from [44]-[46] [78]

$$
\begin{aligned}
\bar{\alpha}_{o} & =\bar{\alpha}_{1}(1 / \phi)+\left(\bar{\alpha}_{2}=\bar{\alpha}_{1} / 2\right)+\bar{\alpha}_{3}+\bar{\alpha}_{4}=60(1 / \phi)+30+9+1=137+k_{o} \\
& =135+\phi^{5}\left(1-\phi^{5}\right)=137.0820393
\end{aligned}
$$

where $\phi=(\sqrt{5}-1) / 2$ and $\bar{\alpha}_{4}=\bar{\alpha}_{Q G}=1$ is the largest possible quantum gravity inverse coupling [41] [44]. The next step in our plasability "derivation" is to notice that $\bar{\alpha}_{1}+\bar{\alpha}_{2}+\bar{\alpha}_{3}+\bar{\alpha}_{4}=100$ and that this sum could be viewed as a normed value for summing over all the infinite dimensions spanning the fractal-Cantorian spacetime of our theory [44]. In other words this 100 is a normed value for the number of internal as well as external dimensions or brocken symmetries. Now we divide these dimension into three categories. First the "visible" dimension, i.e. the 3 space dimension plus the time dimension of our classical daily experience [24]. The second category of dimensions are the compactified 22 left from the bosonic Nambu-Veneziano strong interaction dimension [24]. The third category of dimensions are the diluting rest, i.e. $100-(22+4)=100-26=74$ which represents a finite value for the infinitely many fractal dimensions spanning our fractal spacetime [40]. The next step in our explanation is now quite obvious. We hold it that the various percentages of the energy density of the universe are based on the preceding categorical subdivision of the various normed expectation numbers of the spacetime and internal dimensions [24]. In other words, the four dimensions of space-time correspond to 3 percent ordinary matter and 1 percent ordinary energy and radiation making up 4\% altogether [47]-[51]. The 22 compactified dimensions on the other hand correspond to a 22\% dark, i.e. "compactified" matter [21] [27] [35]. Finally we are left with the well hidden and diluted rest, namely $100-(4+22)=74 \%$ truly pure dark energy responsible for the negative pressure behind the observed accelerated cosmic expansion. Neddless to say these results, taken on face value, are simple integer approximations of the various cosmological measurements of which the majority put ordinary energy at $4.5 \% \cong 4 \%$, dark matter at $22 \%$ and dark energy at $74.5 \cong 74 \%$ [27] [49].

The interesting question on the fundamental level of set theory is to ask how the empty set splits into two sets separating pure dark matter from pure dark energy. Our guess is that it is a very similar phenomenon and analysis to that leading to phase transition from purely ordinary matter to purely ordinary energy [24]. The set theoretical analysis behind the preceding illucidation is currently in progress but we decided to release the present incomplete information in the hope of attracting more thinking in this direction.

\section{Topological Defects, Texture and the Empty Set}

An extremely powerful mathematical subject which benefited cosmology is understanably algebraic topology. Without going into any detail we note the information given in the following Table 1 and add the conjecture that the dimension of texture is -1 and that it could be extrapolated to mean an empty set-like Cantorian wild topology [53] [54] akin to Alexander Horns [38]. Texture in this interpretation corresponds to dark energy and the negative sign to a negative cosmological constant.

Table 1. Cosmic topological defects following the classification of Vilenkin and Shellard [52]. Note that we added the conjecture dim(textures) $=-1$ which in effect equates texture to an empty Cantor set [12].

\begin{tabular}{cc}
\hline Topological defect & Dimension \\
\hline Domain walls & 2 \\
String & 1 \\
Monopoles & 0 \\
Textures & -1 (conjectured) \\
\hline
\end{tabular}




\section{The Fundamental Role of Hardy's Quantum Entanglement}

The importance of the theoretical discovery of Hardy's fundamental probability of a quantum entanglement $\mathrm{P}($ Hardy $)=\phi^{5}$ and its subsequent accurate experimental verification [24] cannot be stressed enough [19] [20] [23]. At a minimum the present work and the understanding of the essence and meaning of dark energy could not be understood in its full ramifications without the quantum entanglement of the cosmos [19]. Without repeating previous arguments and analysis, we just recall for the sake of completeness that $E(O)$ and $E(D)$ could be interpreted and written in terms of Hardy’s quantum entanglement as [15]-[23]

$$
E(O)=P(\text { Hardy })\left(\frac{1}{2} m(v \rightarrow c)^{2}\right)=\left(\phi^{5} / 2\right) m c^{2}
$$

and

$$
E(D)=1-E(O)=\left(5 \phi^{2} / 2\right)\left(m c^{2}\right)
$$

\section{Conclusion}

In a sense we are dealing here with a cosine of the butterfly effect. A small feeble local effect in the form of an attractive gravity induces at infinity an accumulated effect of anti-gravity adjacent to the horizon. In a sense exotic ideas that the universe may resemble a giant black hole or a knot compliment at infinity and therefore neither open nor closed but topologically clopen may not be that far off after all [71] [74]. That way cosmological data and observation collected over a very long period culminating in several deserving Nobel Prizes in Physics has fused various theories together and confirmed the reality of Hawking's radiation, Unruh's temperature, antigravity and Rindler space-time all apart of completing the magnificent work of Einstein's relativity, PlanckBohr-Heisenberg's quantum mechanics and Boltzmann thermodynamics [24] as indicated in the eminent work of T. Padmanabhan and his school [32] [33]. One could of course argue that the present derivation based on an analogy between metal forming, pinching of elastic types and the real behavior of a material space-time micropolar elasticity [76] [78] is less fundamental than previous derivations starting from the zero set as a prequantum particle and the empty set as a pre-quantum wave [36]-[38] [77]. However, this is a largely subjective judgement and a matter of taste and personal philosophical stance [24]. In fact one could view the difference between the negative dimension of the empty set $D_{T}=1$ and the cosmological constant $\Lambda=-1$ [34] as mere mathematical and physical tautology. In conclusion we have to admit that because of space limitation we have hardly touched upon many vital points which could have enhanced understanding the magnificent interconnectivity of mathematics, high energy physics and cosmology leading to the present synthesis. For instance we did not discuss the role of symplectic geometry which would have made the appearance of the golden mean and its derivatives and powers everywhere in our theory plausible, even unavoidable. However the reader may find all these points and more adequately covered in Refs. [55]-[75].

\section{References}

[1] Heisenberg, W. (1969) Der Teil und das Ganze,” Piper, Munich,

[2] Weyl, H. (1923) Raum-Zeit -Materie.Sprin ger, Berlin. http://dx.doi.org/10.1007/978-3-642-98950-6

[3] Hawking, S. and Penrose, R. () The Nature of Space and time. Princeton University Press, New Jersey, 1996.

[4] Weibel, P., Ord, G. and Rössler, O. (2005) Space-Time Physics and Fractality: Festschrift in Honour of Mohamed El Naschie on the Occasion of His 60th Birthday. Springer, Vienna-New York.

[5] Halpern, P. (2004) The Great Beyond. John Wiley, Hoboken.

[6] Ye, F.Y. (2009) From Chaos to Unification: U Theory vs M Theory. Chaos, Solitons \& Fractals, 42, 89-93. http://dx.doi.org/10.1016/j.chaos.2008.10.030

[7] Marek-Crnjac, L. (2013) An Invitation to El Naschie’s Theory of Cantorian Space-Time and Dark Energy. Inter- national Journal of Astronomy and Astrophysics, 3, 464-471. http://dx.doi.org/10.4236/ijaa.2013.34053

[8] He, J.-H. and Marek-Crnjac, L. (2013) The Quintessence of El Naschie’s Theory of Fractal Relativity and Dark Energy. Fractal Space-Time and Non-Commutative Geometry in High Energy Physics, 3, 130-137.

[9] El Naschie, M.S. (2013) Experimentally Based Theoretical Arguments that Unruh’s Temperature, Hawkings’s Vacuum Fluctuation and Rindler’s Wedge Are Physically Real. American Journal of Modern Physics, 2, 357-361. 
[10] Persinger, M. and Koren, S. (2013) Dimensional Analysis of Geometric Products and the Boundary Conditions of the Universe: Implications for Quantitative Value for the Latency to Display Entanglement. The Open Astronomy Journal, 6, 10-13. http://dx.doi.org/10.2174/1874381101306010010

[11] Yang, X.-J., Baleanu, D. and Zhong, W.-P. (2013) Approximate Solutions for Diffusion Equations on Cantor Space-Time,” Proceedings of the Romanian Academy, Series A, 14, 127-133.

[12] Marek-Crnjac, L. (2013) Cantorian Space-Time Theory-The Physics of Empty Sets in Connection With Quantum Entanglement and Dark Energy. Lambert Academic Publishing, Saarbrücken.

[13] El Naschie, M.S. (2007) Transfinite Neoimpressionistic Reality of Quantum Space-Time,” New Advances in Physics, 1, 11-122.

[14] Malinowski, J.J. (2011) Fractal Physics Theory-Foundation. Fundamental Journal of Modern Physics, 1, $133-168$.

[15] El Naschie, M.S. (2013) Nash Embedding of Witten’s M-Theory and Hawking-Hartle Quantum Wave of Dark Energy. Journal of Modern Physics, 4, 1417-1428. http://dx.doi.org/10.4236/jmp.2013.410170

[16] El Naschie, M.S. (2013) From Yang-Mills Photon in Curved Space-Time to Dark Energy Density. Journal of Quantum Information Science, 3, 121-126. http://dx.doi.org/10.4236/jqis.2013.34016

[17] El Naschie, M.S. (2013) A Rindler-KAM Space-Time Geometry and Scaling the Planck Scale Solves Quantum Relativity and Explains Dark Energy. International Journal of Astronomy and Astrophysics, 3, 483-493. http://dx.doi.org/10.4236/ijaa.2013.34056

[18] El Naschie, M.S. (2012) On the Need for Fractal Logic in High Energy Quantum Physics,” International Journal of Modern Nonlinear Theory and Application, 1, 84-92. http://dx.doi.org/10.4236/ijmnta.2012.13012

[19] El Naschie, M.S. (2011) Quantum Entanglement as a Consequence of a Cantorian Micro Space-Time Geometry. Journal of Quantum Information Science, 1, 50-53. http://dx.doi.org/10.4236/jqis.2011.12007

[20] El Naschie, M.S. (2013) A Resolution of Cosmic Dark Energy via a Quantum Entanglement Relativity Theory. Journal of Quantum Information Science, 3, 23-26. http://dx.doi.org/10.4236/jqis.2013.31006

[21] El Naschie, M.S. (2004) A Review of E-Infinity and the Mass Spectrum of High Energy Particle Physics. Chaos, Solitons \& Fractals, 19, 209-236. http://dx.doi.org/10.1016/S0960-0779(03)00278-9

[22] Helal, M.A., Marek-Crnjac, L. and He, J.-H. (2013) The Three Page Guide to the Most Important Results of M.S. El Naschie's Research in E-Infinity and Quantum Physics and Cosmology. Open Journal of Microphysics, 3, 141-145. http://dx.doi.org/10.4236/ojm.2013.34020

[23] El Naschie, M.S. (2013) Quantum Gravity and Dark Energy Using Fractal Planck Scaling. Journal of Modern Physics, 4, 31-38.

[24] Penrose, R. (2004) The Road to Reality. Jonathan Cape, London.

[25] El Naschie, M.S. (1990) Stress, Stability and Chaos in Structural Engineering: An Energy Approach. McGraw-Hill international Editions: Civil Engineering Series, London, Tokyo.

[26] He, J.-H. and Marek-Crnjac, L. (2013) Mohamed El Naschie's revision of Albert Einstein's E = mc ${ }^{2}$ : A Definite Resolution of the Mystery of the Missing Dark Energy of the Cosmos. International Journal of Modern Nonlinear Theory and Application, 2, 55-59. http://dx.doi.org/10.4236/ijmnta.2013.21006

[27] El Naschie, M.S. (2013) A Unified Newtonian-Relativistic Quantum Resolution of the Supposedly Missing Dark Energy of the Cosmos and the Constancy of the Speed of Light. International Journal of Modern Nonlinear Theory and Application, 2, 43-54. http://dx.doi.org/10.4236/ijmnta.2013.21005

[28] Marek-Crnjac, L., et al. (2013) Chaotic Fractal Tiling for the Missing Dark Energy and Veneziano Model. Applicationes Mathematicae, 4, 22-29. http://dx.doi.org/10.4236/am.2013.411A2005

[29] Susskind, L. and Lidesay, J. (2005) The Holographic Universe-An Introduction to Black Holes, Information and the String Theory Revolution. World Scientific, Singapore.

[30] Ellis, G.F.R. and Williams, R.M. (2000) Flat and Curved Space-Time. Oxford University Press, Oxford.

[31] Bronshtein, I.N. and Semendyayev, K.A. (1985) Handbook of Mathematics. Van Nostrand Reinhold Company, New York.

[32] Padmanabhan, T. (2004) Dark Energy: The Cosmological Challenge of the Millennium. arXiv: astro-ph/0411044V1

[33] Padmanabhan, T. (2003) Gravity from Space-Time Thermodynamics,” Astrophysics and Space Science, 285, $407-417$. http://dx.doi.org/10.1023/A:1025448712533

[34] El Naschie, M.S. (2013) Topological-Geometrical and Physical Interpretation of the Dark Energy of the Cosmos as a “Halo” Energy of the Schrödinger Quantum Wave,” Journal of Modern Physics, 4, 591-596. http://dx.doi.org/10.4236/jmp.2013.45084

[35] El Naschie, M.S. (2013) What Is the Missing Dark Energy in a Nutshell and the Hawking-Hartle Quantum Wave Col- 
lapse. International Journal of Astronomy and Astrophysics, 3, 205-211. http://dx.doi.org/10.4236/ijaa.2013.33024

[36] El Naschie, M.S. and Helal, A. (2013) Dark Energy Explained via the Hawking-Hartle Quantum Wave and the Topology of Cosmic Crystallography. International Journal of Astronomy and Astrophysics, 3, 318-343. http://dx.doi.org/10.4236/ijaa.2013.33037

[37] El Naschie, M.S. (2008) Adic Unification of the Fundamental Forces and the Standard Model. Chaos, Solitons \& Fractals, 38, 1011-1012. http://dx.doi.org/10.1016/j.chaos.2008.04.047

[38] El Naschie, M.S. (2006) Advanced Prerequisites for E-Infinity. Chaos, Solitons \& Fractals, 30, 636-641. http://dx.doi.org/10.1016/j.chaos.2006.04.044

[39] El Naschie, M.S. (2004) Quantum Gravity from Descriptive Set Theory. Chaos, Solitons \& Fractals, 19, $1339-1344$. http://dx.doi.org/10.1016/i.chaos.2003.08.009

[40] El Naschie, M.S. (2004) Quantum Gravity from Descriptive Set Theory. Chaos, Solitons \& Fractals, 19, $1339-1344$. http://dx.doi.org/10.1016/j.chaos.2003.08.009

[41] El Naschie, M.S. (2007) A Review of Application and Results of E-Infinity Theory. International Journal of Nonlinear Sciences and Numerical Simulation, 8, 11-20. http://dx.doi.org/10.1515/IJNSNS.2007.8.1.11

[42] Vladimirov, V., Valovich, I. and Zelenov, E. (1994) P-Adic Analysis and Mathematical Physics. World Scientific, Singapore. http://dx.doi.org/10.1142/1581

[43] Khrennikov, A. (1997) Non-Archimedean Analysis: Quantum Paradoxes, Dynamical Systems and Biological Methods. Kluwer Academic Publishers, Dordrecht. http://dx.doi.org/10.1007/978-94-009-1483-4

[44] El Naschie, M.S. (2009) The Theory of Cantorian Space-Time and High Energy Particle Physics (An Informal Review). Chaos, Solitons \& Fractals, 41, 2635-2646. http://dx.doi.org/10.1016/j.chaos.2008.09.059

[45] El Naschie, M.S. (2006) Elementary Prerequisites for E-Infinity (Recommended Background Readings in Nonlinear Dynamics, Geometry and Topology). Chaos, Solitons \& Fractals, 30, 579-605. http://dx.doi.org/10.1016/j.chaos.2006.03.030

[46] El Naschie, M.S. (2004) The Concepts of E-Infinity: An Elementary Introduction to the Cantorian-Fractal Theory of Quantum Physics. Chaos, Solitons \& Fractals, 22, 495-511. http://dx.doi.org/10.1016/j.chaos.2004.02.028

[47] Ruiz-Lapuente, P. (2010) Dark Energy. Cambridge University Press, Cambridge.

[48] Perkins, D. (2009) Particle Astrophysics. 2nd Edition, Oxford University Press, Oxford.

[49] Amendola, L. and Tsujikawa, S. (2010) Dark Energy-Theory and Observations. Cambridge University Press, Cambridge. http://dx.doi.org/10.1017/CBO9780511750823

[50] Bahcall, J., Pivan, T. and Weinberg, S. (2004) Dark Matter in the Universe. 2nd Edition, World Scientific, Singapore.

[51] Weinberg, S. (2008) Cosmology. Oxford University Press, Oxford.

[52] Vilenkin, A. and Shellard, E.S. (1994) Cosmic Strings and Other Topological Defects. Cambridge University Press, Cambridge.

[53] El Naschie, M.S. (2002) Wild Topology, Hyperbolic Geometry and Fusion Algebra of High Energy Particle Physics. Chaos, Solitons \& Fractals, 13, 1935-1945. http://dx.doi.org/10.1016/S0960-0779(01)00242-9

[54] El Naschie, M.S. (2002) Quantum Loops, Wild Topology and Fat Cantor Sets in Transfinite High Energy Physics. Chaos, Solitons \& Fractals, 13, 1167-1174. http://dx.doi.org/10.1016/S0960-0779(01)00210-7

[55] Tao, Y. (2013) The Validity of Dimensional Regularization Method on Fractal Space-Time. Journal of Applied Mathematics, 2013, Article ID: 308691. http://dx.doi.org/10.1155/2013/308691

[56] Marek-Crnjac, L. (2009) A Feynman Path Integral-Like Method for Deriving the Four Dimensionality of Space-Time from First Principles. Chaos, Solitons \& Fractals, 41, 2471-2473. http://dx.doi.org/10.1016/j.chaos.2008.09.014

[57] El Naschie, M.S. (2002) Determining the Temperature of the Microwave Background Radiation from the Topology and Geometry of Space-Time. Chaos, Solitons \& Fractals, 14, 1121-1126. http://dx.doi.org/10.1016/S0960-0779(02)00172-8

[58] El Naschie, M.S. (1997) A Note on Quantum Gravity and Cantorian Space-Time. Chaos, Solitons \& Fractals, 8, 131133. http://dx.doi.org/10.1016/S0960-0779(96)00128-2

[59] El Naschie, M.S. (1998) Superstrings, Knots and Non-Commutative Geometry in E-Infinity Space. International Journal of Theoretical Physics, 37, 2935-2951. http://dx.doi.org/10.1023/A:1026679628582

[60] Lehmann, T. (1966) Formänderung Eines Klassischen Kontinuums in Vierdimensionaler Darstellung. In: Görtler, H., Ed., Applied Mechanics, Springer-Verlag, Heidelberg, 376-382.

[61] Mukhamedov, A.M. (2007) E-Infinity as a Fiber Bundle and Its Theormodynamics. Chaos, Solitons \& Fractals, 33, 717-724. http://dx.doi.org/10.1016/j.chaos.2006.11.016 
[62] El Naschie, M.S. (1979) Die Ableitung Einer Konsistenten Schalentheorie in Dem Dreidimensionalen Kontinuum. Österreichische Ingenieur-Zeitschrift (Austrian Engineering Journal), 22, 339-344.

[63] El Naschie, M.S. (2009) Curvature, Lagrangian and Holonomy of Cantorian-Fractal Space-Time. Chaos, Solitons \& Fractals, 41, 2163-2167. http://dx.doi.org/10.1016/i.chaos.2008.08.015

[64] El Naschie, M.S. (2009) An Irreducibly Simple Derivation of the Hausdorff Dimension of Space-Time. Chaos, Solitons \& Fractals, 41, 1902-1904. http://dx.doi.org/10.1016/j.chaos.2008.07.043

[65] El Naschie, M.S. (1999) Quantum Groups and Hamiltonian Sets on a Nuclear Space-Time Cantorian Manifold. Chaos, Solitons \& Fractals, 10, 1251-1256. http://dx.doi.org/10.1016/S0960-0779(99)00009-0

[66] El Naschie, M.S. (2004) The Symplectic Vacuum, Exotic Quasi Particles and Gravitational Instantons. Chaos, Solitons \& Fractals, 22, 1-11. http://dx.doi.org/10.1016/j.chaos.2004.01.015

[67] El Naschie, M.S. (2008) Transfinite Harmonization by Taking the Dissonance out of the Quantum Field Symphony. Chaos, Solitons \& Fractals, 36, 781-786. http://dx.doi.org/10.1016/j.chaos.2007.09.018

[68] Vrobel, S. (2011) Fractal Time. World Scientific, Singapore.

[69] El Naschie, M.S. (2012) The Minus One Connection of Relativity, Quantum Mechanics and Set Theory. Fractal Space-Time and Non-Commutative Geometry in High Energy Physics, 2, 131-134.

[70] El Naschie, M.S. and Olsen, S. (2011) When Zero Is Equal to One-A Set Theoretical Resolution of Quantum Paradoxes. Fractal Space-Time and Non-Commutative Geometry in High Energy Physics, 1, 11-24.

[71] El Naschie, M.S. (2013) The Quantum Gravity Immirzi Parameter-A General Physical and Topological Interpretation. Gravitation and Cosmology, 19, 151-155. http://dx.doi.org/10.1134/S0202289313030031

[72] El Naschie, M.S. (2013) Determining the Missing Dark Energy of the Cosmos from a Light Cone Exact Relativistic Analysis. Journal of Physics, 2, 18-23.

[73] Adams, C.C. (1994) The Knot Book. H. Freeman, New York, 244-246.

[74] Pathvia, R.K. (1972) The Universe as a Black Hole. Nature, 240, 298-299. http://dx.doi.org/10.1038/240298a0

[75] Nesteruk, A. (2013) Physics in Christianity. In: Runehov, A. and Oviedo, L., Eds., Encyclopedia of Sciences and Religion, 3, 1718-1729.

[76] Watson, A., Reid, S., Johnson, W. and Thomas, S. (1976) Large Deformations of Thin-Walled Circular Tubes under Transverse Loading-II. International Journal of Mechanical Sciences, 18, 389-397. http://dx.doi.org/10.1016/0020-7403(76)90015-1

[77] Marek-Crnjac, L. (2012) Quantum Gravity in Cantorian Space-Time. In: Soberio, R., Ed., Quantum Gravity, INTECH Publishing, 87-100. www.intechopen.com.

[78] Cosserat, E. and Cosserat, F. (1909) Théorie des Corps déformables. Hermann, Paris.

[79] El Naschie, M.S. (2013) The Hyperbolic Extension of Sigalotti-Heni-Sharifzadeh’s Golden Triangle of Special Theory of Relativity and the Nature of Dark Energy. Journal of Modern Physics, 4, 354-356. http://dx.doi.org/10.4236/jmp.2013.43049 